\title{
HEEGAARD SPLITTINGS OF SEIFERT FIBERED SPACES WITH BOUNDARY
}

\author{
JENNIFER SCHULTENS
}

\begin{abstract}
We give the classification theorem for Heegaard splittings of fiberwise orientable Seifert fibered spaces with nonempty boundary. A thin position argument yields a reducibility result which, by induction, shows that all Heegaard splittings of such manifolds are vertical in the sense of Lustig-Moriah. Algebraic arguments allow a classification of the vertical Heegaard splittings.
\end{abstract}

\section{INTRODUCTION}

Seifert manifolds were the first 3-manifolds known to possess distinct Heegaard splittings. In [8] Moriah and later in [1] Boileau, Collins and Zieschang described distinct Heegaard splittings of Seifert manifolds which fiber over $S^{2}$ and possess three exceptional fibers. In particular, they described two structurally different types of Heegaard splittings for these manifolds: vertical Heegaard splittings and horizontal Heegaard splittings. We here extend the notion of a vertical Heegaard splitting to arbitrary Seifert manifolds and prove the following:

Theorem 4.2. An irreducible Heegaard splitting of a fiberwise orientable Seifert manifold with nonempty boundary is vertical.

In most cases, Theorem 4.2 establishes the complete classification of Heegaard splittings for the manifolds in question. Since their vertical Heegaard splittings may be classified using work of Lustig [6] and of Lustig-Moriah [7].

Theorem 5.1. Let $M$ be a fiberwise orientable Seifert fibered space with nonempty boundary and with Seifert invariants $\left\{g, e ; \alpha_{1}, \beta_{1}, \ldots, \alpha_{k}, \beta_{k}\right\}$. Let $\beta=\left\{i \mid \beta_{i} \neq \pm 1\left(\bmod \alpha_{i}\right) ; i=1, \ldots, k\right\}$. Then the two vertical Heegaard splittings $F\left(i_{1}, \ldots, i_{n} ; j_{1}, \ldots, j_{l}\right)$ and $F\left(\zeta_{1}, \ldots, \zeta_{\nu} ; j_{1}, \ldots, j_{l}\right)$ are isotopic if and only if $\beta \cap\left\{i_{1}, \ldots, i_{n}\right\}=\beta \cap\left\{\zeta_{1}, \ldots, \zeta_{\nu}\right\}$.

We review definitions of compression bodies and Heegaard splittings (cf. [11]).

Definitions. For $M$ a manifold, denote the boundary of $M$ by $\partial M$. For $S$ a submanifold of $M$, denote a closed regular neighborhood of $S$ in $M$ by $N(S)$ and denote an open regular neighborhood of $S$ in $M$ by $\eta(S)$.

Received by the editors September 30, 1993 and, in revised form, September 19, 1994.

1991 Mathematics Subject Classification. Primary 57N10.

Research supported by a University of California President's Dissertation Year Fellowship. 
A compression body $\mathrm{W}$ is a 3-manifold which may be constructed by adding 2handles to a (surface) $\times I$ along a collection of disjoint simple closed curves on (surface) $\times\{0\}$, and capping off any resulting 2-sphere boundary components with 3-balls. The component (surface) $\times\{1\}$ of $\partial \mathrm{W}$ is denoted by $\partial_{+} W$ and the surface $\partial W-\partial_{+} W$, which may or may not be connected, is denoted by $\partial_{-} W$. If $\partial_{-} W=\varnothing$, then $W$ is a handlebody. If $W=\partial_{+} W \times I, W$ is called a trivial compression body.

A Heegaard splitting of $M$ is a pair $\left(W_{1}, W_{2}\right)$ of compression bodies, such that $M=W_{1} \cup W_{2}$ and $W_{1} \cap W_{2}=\partial_{+} W_{1}=\partial_{+} W_{2}=F$, for some connected closed orientable surface $F$. This $F$ is called the splitting surface of $\left(W_{1}, W_{2}\right)$. Two Heegaard splittings of $M$ are considered equivalent, if their splitting surfaces are isotopic. A Heegaard splitting is reducible if there exists an essential simple closed curve $c \subset F$ that bounds embedded disks in both $W_{1}$ and $W_{2}$. A Heegaard splitting is irreducible if it is not reducible. A stabilization of $\left(W_{1}, W_{2}\right)$ is a Heegaard splitting which is obtained by taking the connected sum of pairs of $\left(M^{3}, W_{1}\right)$ and a finite number of $\left(S^{3}, T\right)$ summands, where $T$ is the standard unknotted torus in $S^{3}$. Note that a Heegaard splitting of an irreducible manifold is reducible if and only if it is a stabilization [3, Haken's theorem].

By Epstein's Theorem, a Seifert fibered space is a compact 3-manifold that admits a foliation into circles. We call this foliation a Seifert fibration and we call the leaves of the foliation the fibers of the Seifert fibration. If we identify each fiber to a point, we obtain a surface $P$, called the base of the Seifert fibration. Note that in general, the natural projection map $p: M \rightarrow P$ does not define a fiber bundle in the usual sense, unless we exclude a finite number of points $x_{1}, \ldots, x_{k}$ of $P$ and the corresponding fibers $e_{1}, \ldots, e_{k}$ of $M$. The points $x_{1}, \ldots, x_{k}$ are called exceptional points and the fibers $e_{1}, \ldots, e_{k}$ are called exceptional fibers. We will denote $e_{1} \cup \ldots \cup e_{k}$ by $E$.

Let $D_{i}$ be a closed regular neighborhood of $x_{i}$. Then $V_{i}=p^{-1}\left(D_{i}\right)$ is a solid torus. This $V_{i}$ is itself a Seifert fibered space, called a fibered solid torus. Note that, in general, the fibration of $V_{i}$ is not the product fibration (in which fibers would be point $\} \times S^{1}$ ).

We will call $M$ a fiberwise orientable Seifert fibered space, if both $M$ and $P$ are orientable. To a fiberwise orientable Seifert fibered space $M$ there is associated a set of invariants $\left\{g, e ; \alpha_{1}, \beta_{1}, \ldots, \alpha_{k}, \beta_{k}\right\}$. The invariant $g$ denotes the genus of $P$. The invariants $\left(\alpha_{i}, \beta_{i}\right)$ are associated to the exceptional fiber $e_{i}$. They are determined by the fibered solid torus $V_{i}$. Indeed, if $V_{i}$ is fibered by $(p, q)$ torus knots, then we set $\alpha_{i}=p$ and require that $0<\beta_{i} \leq \alpha_{i}$ and that $\beta_{i} \cdot q=1 \bmod p$. The rational Euler number $e$ eliminates any resulting ambiguity. For a definition of $e$ and more detail see [13].

Set $S=$ closure $\left(P-\left(D_{1} \cup \ldots \cup D_{k}\right)\right)$. Then $M-\left(V_{1} \cup \ldots \cup V_{k}\right)$ is an ordinary circle bundle over the surface $S$. Since $P$ is orientable and has nonempty boundary, $H^{2}\left(S, \cup\left(\partial D_{i}\right)\right)$ is trivial. Thus the Euler class of the bundle $M-$ $\left(V_{1} \cup \ldots \cup V_{k}\right)$ over $P-\left(D_{1} \cup \ldots \cup D_{k}\right)$ is trivial and hence this bundle is trivial. In particular, if $M$ has no exceptional fibers, then $M=S \times S^{1}$, and $S$ is a compact orientable surface.

We construct vertical Heegaard splittings for $M$. This definition extends that of Lustig and Moriah [7] to the case of compact fiberwise orientable Seifert manifolds. 


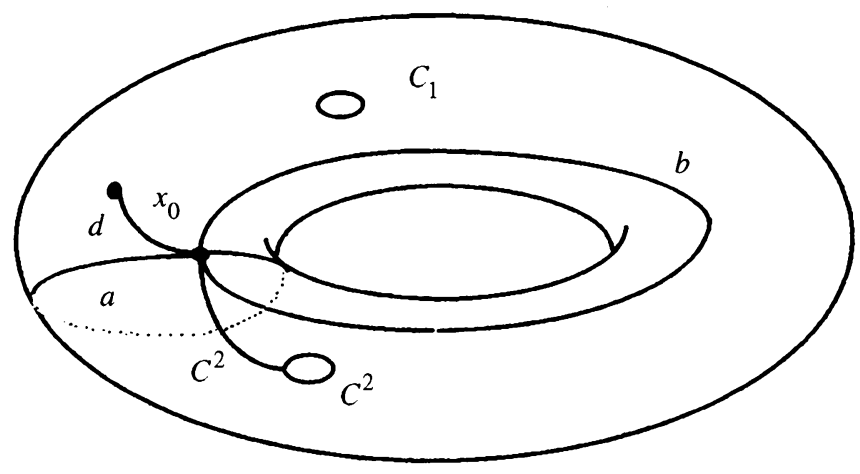

FIGURE 1.1. A collection of arcs defining vertical Heegaard splittings

Roughly speaking, vertical Heegaard splittings are those for which each exceptional fiber appears as the core of a 1-handle in one of the compression bodies. In order to distinguish the various vertical splittings we need to be more precise. Consider a copy of $S$, together with all the curves here defined, in $M$. Denote the components of $\partial S-\left(\partial D_{1} \cup \ldots \cup \partial D_{k}\right)$ by $C^{1}, \ldots, C^{m}$. Let $x_{0}$ be a point in $\operatorname{int}(S)$. For $j=2, \ldots, m$, let $c^{j}$ be a simple arc in $\mathrm{S}$ connecting $x_{0}$ to $C^{j}$. For $i=1, \ldots, k$, let $d_{i}$ be an arc connecting $x_{0}$ to $\partial D_{j}$. Let $c=c^{2} \cup \ldots \cup c^{m}$ and let $d=d_{1} \cup \ldots \cup d_{k}$. Further, let $a_{1}, b_{1}, \ldots, a_{g}, b_{g}$ be a collection of arcs based at $x_{0}$ which cuts $S$ into a disk with punctures corresponding to $C^{1}, \ldots, C^{m}, \partial D_{1}, \ldots, \partial D_{k}$. We may assume that all arcs chosen are disjoint except at $x_{0}$. See Figure 1.1.

Let $\left\{i_{1}, \ldots, i_{n}\right\} \subset\{1, \ldots, k\}$ be a collection of distinct indices (for the exceptional fibers) and denote by $\left\{l_{1}, \ldots, l_{k-n}\right\}$ the elements of $\{1, \ldots, k\}-$ $\left\{i_{1}, \ldots, i_{n}\right\}$. Let $\left\{j_{1}, \ldots, j_{l}\right\} \subset\{2, \ldots, m\}$ be another collection of distinct indices (for the boundary components) and denote by $\left\{k_{1}, \ldots, k_{m-l-1}\right\}$ the elements of $\{2, \ldots, m\}-\left\{j_{1}, \ldots, j_{l}\right\}$. Assume that either $n \neq 0$ or $l \neq 0$. Let

$$
\Omega\left(i_{1}, \ldots, i_{n} ; j_{1}, \ldots, j_{l}\right)
$$

be the graph obtained from

$$
a_{1} \cup b_{1} \cup \ldots \cup a_{g} \cup b_{g} \cup d \cup \partial D_{l_{1}} \cup \ldots \cup \partial D_{l_{k-n}} \cup c \cup C^{k_{1}} \cup \ldots \cup C^{k_{m-l-1}}
$$

by modifying

$$
\begin{aligned}
& d_{l_{1}} \cup \ldots \cup d_{l_{k-n}} \cup \partial D_{l_{1}} \cup \ldots \cup \partial D_{l_{k-n}} \\
& \cup c_{k_{1}} \cup \ldots \cup c_{k_{m-l-1}} \cup C^{k_{1}} \cup \ldots \cup C^{k_{m-l-1}}
\end{aligned}
$$

so that they lie in interior $(S)$. And set

$$
\begin{aligned}
& W_{1}\left(i_{1}, \ldots, i_{n} ; j_{1}, \ldots, j_{l}\right) \\
& \quad=N\left(\Omega\left(i_{1}, \ldots, i_{n} ; j_{1}, \ldots, j_{l}\right)\right. \\
& \left.\qquad V_{i_{1}} \cup \ldots \cup V_{i_{n}} \cup\left(C^{j_{1}} \times S^{1}\right) \cup \ldots \cup\left(C^{j_{l}} \times S^{1}\right)\right) .
\end{aligned}
$$

Set

And set

$$
W_{2}=\operatorname{closure}\left(M-W_{1}\right) .
$$




$$
\begin{aligned}
& F\left(i_{1}, \ldots, i_{n} ; j_{1}, \ldots, j_{l}\right) \\
& \quad=W_{1}\left(i_{1}, \ldots, i_{n} ; j_{1}, \ldots, j_{l}\right) \cap W_{2}\left(i_{1}, \ldots, i_{n} ; j_{1}, \ldots, j_{l}\right) \\
& \quad=\partial_{+} W_{1}\left(i_{1}, \ldots, i_{n} ; j_{1}, \ldots, j_{l}\right) \\
& \quad=\partial_{+} W_{2}\left(i_{1}, \ldots, i_{n} ; j_{1}, \ldots, j_{l}\right) .
\end{aligned}
$$

Clearly $W_{1}\left(i_{1}, \ldots, i_{n} ; j_{1}, \ldots, j_{l}\right)$ is a compression body. To see that $W_{2}\left(i_{1}, \ldots, i_{n} ; j_{1}, \ldots, j_{l}\right)$ is a compression body, for instance in the case when $n \neq 0$, isotope $x_{0}$ to lie on $e_{i_{1}}$, then the arcs $a_{i}, b_{i}, d_{i_{j}}, c^{j_{i}}$,

$$
\left(\partial\left(\operatorname{bicollar}\left(d_{l_{j}}\right) \cup \partial D_{l_{j}}\right)\right)-\left(\operatorname{bicollar}\left(d_{l_{j}} \cap \partial D_{l_{j}}\right)\right),
$$

and

$$
\left(\partial\left(\operatorname{bicollar}\left(c^{j_{i}}\right) \cup C^{j_{i}}\right)\right)-\left(\operatorname{bicollar}\left(c^{j_{i}}\right) \cap C^{j_{i}}\right)
$$

define 2-handles in $W_{2}$ (e.g., $\left.\left(a_{1} \times S^{1}\right)-\left(\left(x_{0} \times S^{1}\right) \cup a_{1}\right)\right)$. We can construct $W_{2}$ by attaching these 2 -handles to $F\left(i_{1}, \ldots, i_{n} ; j_{1}, \ldots, j_{l}\right)$ and capping off the resulting 2-sphere boundary component with a 3-ball. It follows that

$$
\left(W_{1}\left(i_{1}, \ldots, i_{n} ; j_{1}, \ldots, j_{l}\right), W_{2}\left(i_{1}, \ldots, i_{n} ; j_{1}, \ldots, j_{l}\right)\right)
$$

is a Heegaard splitting with splitting surface

$$
F\left(i_{1}, \ldots, i_{n} ; j_{1}, \ldots, j_{l}\right) .
$$

Any Heegaard splitting for $M$ obtained in this way is called a vertical Heegaard splitting.

When $n$ and $l$ are both $0, W_{1}(\varnothing, \varnothing)$ is still a compression body (a handlebody, in fact) in the construction above, but $W_{2}(\varnothing, \varnothing)$ is not. We may correct this mishap by adjoining $x_{0} \times S^{1}$ to $\Omega(\varnothing, \varnothing)$, but it will be shown in 2.3 that the resulting Heegaard splitting is reducible for the manifolds under consideration. We denote the splitting surface obtained in this modified construction by $F(\varnothing, \varnothing)$.

To see that $F\left(i_{1}, \ldots, i_{n} ; j_{1}, \ldots, j_{l}\right)$ is well-defined, apply [12, Theorem 3.1] to $F\left(i_{1}, \ldots, i_{n} ; j_{1}, \ldots, j_{l}\right)$ regarded as a Heegaard splitting of $M-\eta(E)$. In the case where $E=\varnothing$, these Heegaard splittings are also called standard Heegaard splittings of $P \times S^{1}$ rel $\left\{C^{j_{1}}, \ldots, C^{j_{l}}\right\}$.

Note that by forbidding $j_{1}, \ldots, j_{l}$ to be 1 , we have fixed $W_{2}$ as the compression body containing $C^{1}$. Also note that the genus of a vertical Heegaard splitting is $2 g+k+m-1$. The genus of $F(\varnothing, \varnothing)$ is $2 g+k+m$. When $E=\varnothing$, the standard Heegaard splitting of $P \times S^{1}=S \times S^{1}$ rel $\varnothing$ is in fact irreducible.

The proof of the main theorem relies on an induction argument on the number of exceptional fibers of $M$. Theorem 1.1 provides the first step in this induction argument.

Theorem 1.1. For manifolds which are homeomorphic to (compact orientable surface $) \times S^{1}$ all Heegaard splittings are stabilizations of standard Heegaard splittings.

Proof. This is [12, Theorem 5.7]. 
Theorem 1.1 has interesting consequences in the setting of Seifert fibered spaces.

Proposition 1.2. A Heegaard splitting $\left(W_{1}, W_{2}\right)$ of a compact fiberwise orientable Seifert manifold $M$ is vertical if and only if, after isotopy, $\left(W_{1}-\eta(E), W_{2}-\right.$ $\eta(E))$ is a standard Heegaard splitting of $M-\eta(E)$.

Proof. This follows from the construction. More specifically, denote by $C^{m+i_{1}}$, $\ldots, C^{m+i_{n}}$ the components of $\partial(M-\eta(E))$ arising from $e_{i_{1}}, \ldots, e_{i_{n}}$, that is, set $C^{m+i_{1}}=\partial\left(M-\eta\left(e_{i_{1}}\right)\right)-\partial(M), \ldots, C^{m+i_{n}}=\partial\left(M-\eta\left(e_{i_{n}}\right)\right)-$ $\partial(M)$. The splitting surface $F\left(i_{1}, \ldots, i_{n} ; j_{1}, \ldots, j_{l}\right)$ of the vertical Heegaard splitting $\left(W_{1}\left(i_{1}, \ldots, i_{n} ; j_{1}, \ldots, j_{l}\right), W_{2}\left(i_{1}, \ldots, i_{n} ; j_{1}, \ldots, j_{l}\right)\right)$ of $M$ is, after isotopy, the splitting surface of the standard Heegaard splitting of $M$ $\eta(E)$ rel $\left\{C^{j_{1}}, \ldots, C^{j_{l}}, C^{m+i_{1}}, \ldots, C^{m+i_{n}}\right\}$ and vice versa.

Proposition 1.3. If $M$ is a Seifert fibered space with a Heegaard splitting formed by the amalgamation (in the sense used in [12]) of vertical Heegaard splittings of Seifert fibered submanifolds along saturated tori, then this Heegaard splitting is vertical.

Proof. This follows from Proposition 1.2 together with [12, Proposition 2.10].

The proof of the Classification Theorem for Heegaard splittings of Seifert manifolds with boundary falls into four parts. In section 2 , we define a reducibility property used in the proof of the Main Theorem and give a sufficient criterion for its occurrence. Section 3 provides an elementary technical condition which helps guarantee this criterion. In section 4 , we prove the Main Theorem, which says that all irreducible Heegaard splittings of the manifolds under consideration are vertical. In section 5 , we provide a classification of the vertical Heegaard splittings via an algebraic invariant.

I wish to thank Martin Scharlemann for numerous helpful discussions.

\section{REDUCIBILITY}

In this section we introduce a reducibility property which enables the inductive step in the proof of the main theorem.

Definition. Let $e \epsilon E$. If, after isotopy, $F$ is a splitting surface for Heegaard splittings both of $M$ and of $M-\eta(e)$, then we say that $F$ is vertically reducible at $e$.

Lemma 2.2 will give sufficient conditions for $F$ to be vertically reducible at $e$.

Lemma 2.1 (The vacuum lemma). Suppose $F$ is the splitting surface of a Heegaard splitting of the 3-manifold $M, D$ is an essential disk in $W_{1}$ and $\gamma$ is a simple closed curve in $F$ which intersects $\partial D$ once transversely. Then after $\gamma$ is isotoped to lie entirely in interior $\left(W_{1}\right), F$ is also a splitting surface of $M-\eta(\gamma)$. See Figure 2.1.

This lemma owes its illustrative name to the following visualization of its proof: The 1-handle dual to the disk $D$ is dragged along $\gamma(\gamma$ is vacuumed onto the 1-handle through the attaching disk D of the 1-handle). After this procedure $\gamma$ lies entirely on the 1-handle; furthermore, $\gamma$ lies parallel to the 


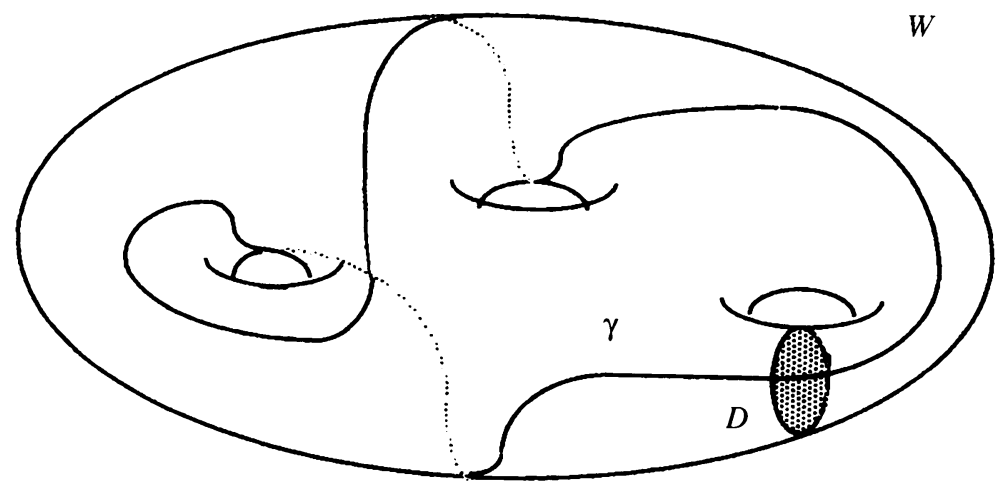

Figure 2.1. A curve $\gamma$ and a disk $D$ in a compression body $W$.

core of the 1-handle. Drilling out the core of a 1-handle in a compression body produces another compression body.

The following proof of Lemma 2.1 was suggested by Geoffrey Mess during the presentation of a more complicated argument.

Proof. Let $N(\gamma)$ be a collar neighborhood of $\gamma$ in $W_{1}$ and let $N(D)$ be a bicollar of $D$ in $W_{1}$. Then $T=N(\gamma) \cup N(D)$ is a solid torus summand of $W_{1}$. We may split $T$ from $W_{1}$ at the disk

$$
D^{\prime}=\operatorname{closure}\left[\partial T-\partial_{+} W\right] \text {. }
$$

We may expand the collection $\left\{D, D^{\prime}\right\}$ of boundary reducing disks of $W_{1}$ to a defining set of disks $\Delta$ of $W_{1}$. Set $\Delta(T)=\{D \in \Delta \mid D \subset T\}$. Now $\Delta-\Delta(T)$ is a defining set of disks for $W_{1}-T$. Isotope $\gamma$ to lie in interior $(\gamma)$ and let $\eta(\gamma)$ be a small regular neighborhood of $\gamma$ which lies in interior $(N(\gamma))$. Then the collection $(\Delta-\Delta T) \cup\left\{D^{\prime}\right\}$ is a defining set of disks for $W_{1}-\eta(\gamma)$.

Lemma 2.2. Suppose $F$ is a splitting surface of a Heegaard splitting for $M$. Suppose further, that $e \in E$ lies in $W_{1}$ and is parallel to a simple closed curve $\gamma$ in $F$ which intersects an essential disk $D$ in $W_{1}$ once transversely. Then, after $e$ is isotoped to lie in interior $\left(W_{1}\right), F$ is also a Heegaard splitting for $M-\eta(e)$, so $F$ is vertically reducible at $e$.

Proof. Let $A$ be the immersed annulus defined by the isotopy of $\gamma$ to $e$. Since $e$ and $\gamma$ are disjoint and simple, $\partial A$ is embedded. Thus we may assume that $A$ is embedded. Then the lemma follows by replacing $N(\gamma)$ by $N(A)$ in the proof of Lemma 2.1 .

The following lemma will establish the interesting fact that, for the manifolds under consideration, $F(\varnothing, \varnothing)$ is reducible and should hence not be counted among their vertical Heegaard splittings.

Lemma 2.3. For $E \neq \varnothing, F(\varnothing, \varnothing)$ is reducible.

Proof. Recall the notation used in defining vertical Heegaard splittings. The arc $\delta=\left(\partial\right.$ (bicollar $\left.\left.\left(d_{1}\right)\right) \cup \partial D_{1}\right)-\left(\right.$ bicollar $\left.\left(d_{1}\right) \cap \partial D_{1}\right)$ defines a disk $\tilde{D}$ in $W_{2}\left(\right.$ take $\left.\left(\delta \times S^{1}\right)-\left(\delta \cup\left(x_{0} \times S^{1}\right)\right)\right)$. Let $H$ be the genus 2 subhandlebody of $W_{1}(\varnothing, \varnothing)$ defined by $\left(x_{0} \times S^{1}\right) \cup \delta$. Let $T=\partial V_{1}$ 
( $=\delta \times S^{1}$.) Then $H$ may be viewed as (punctured $T$ ) $\times I$, with puncture corresponding to $\tilde{D}$.

Let $\Delta_{2}$ be the compressing disk for $W_{2}$ defined by a meridian of $V_{1}$. Since $\Delta_{2}$ is nonseparating in $V_{1}, \partial \Delta_{2}$ defines a nonseparating closed curve in $T \subset$ $\partial V_{1}$. There is a proper arc $\alpha$ in $T$ which intersects this closed curve on $T$ exactly once. This $\alpha$ defines an essential disk $\Delta_{1}$ in $H$ whose boundary intersects that of $\Delta_{2}$ exactly once. The pair $\left(\Delta_{1}, \Delta_{2}\right)$ shows that $F$ is reducible.

\section{MORSE FUNCTIONS AND OUTERMOST ESSENTIAL SADDLES}

Heegaard splittings correspond to Morse functions. Let $\left(W_{1}, W_{2}\right)$ be a Heegaard splitting of the 3-manifold $M$. Then $W_{1}=\left(\left(\partial_{-} W_{1} \times I\right) \cup\right.$ (0-handles)) $\cup$ (1-handles), $W_{2}=\left(\partial_{+} W_{2} \times I\right) \cup$ (2-handles) $\cup$ (3-handles), so $W_{1} \cup W_{2}$ $=\left(\left(\partial_{-} W_{1} \times I\right) \cup(0\right.$-handles $\left.)\right) \cup$ (1-handles) $\cup$ (2-handles) $\cup$ (3-handles) gives a handlebody description of $M$. Excess 0 -handles can be canceled with 1-handles and (dually) excess 3-handles with 2-handles, after which there is at most one 0-handle (precisely when $W_{1}$ is a handlebody), and at most one 3handle (precisely when $W_{2}$ is a handlebody). This handlebody description can be used to define a Morse function $h$ on $M$ (apply [9, Theorem 3.12] repeatedly;. Call $h$ a Morse function induced by $\left(W_{1}, W_{2}\right)$. Here $h$ can be taken to have singular values $0<a_{1}<\ldots<a_{m}<b_{1}<\ldots<b_{n}<1$, with the critical point at level $a_{i}, i=1, \ldots, m$, the center of a 1-handle and the critical point at level $b_{j}, j=1, \ldots, n$, the center of a 2 -handle. For the converse, constructing a Heegaard splitting from a Morse function, see [10, I.3].

Definitions. Let $Q \subset M$ be an immersed compact surface. Let $h: Q \rightarrow[0,1]$ be a Morse function and let $r$ be fixed. An outermost inessential arc $\alpha$ in $h^{-1}(r) \cap Q$ cuts off a disk $D$; the arc $\alpha$ is called a high arc and $D$ a lower disk if for all $x$ in $D-\alpha, h(x)<r=h(\alpha) ; \alpha$ is called a low arc and $D$ an upper disk if for all $x$ in $D-\alpha, h(x)>r=h(\alpha)$.

Let $\Phi_{Q}$ be the singular foliation of a compact surface $Q$ by level sets of $h$. Let $\sigma$ be a leaf in $\Phi_{Q}$ containing a saddle singularity. By Morse general position, $\sigma$ contains one critical point $x$, the saddle point. If $x$ lies in the interior of $Q$ there will be four subarcs of $\sigma$, called separatrices, emanating from $x$. The separatrices may end on $\partial Q$ or may join so that either one pair or two pairs form circles. If a circle formed by separatrices is inessential, then $\sigma$ is called an inessential saddle. Otherwise $\sigma$ is an essential saddle. If $x$ lies on $\partial Q$, there are two subarcs emanating from $x$, again called separatrices. In this case $\sigma$ is called a half-saddle. If the two separatrices join to form a circle which is inessential, then $\sigma$ is called an inessential half-saddle. Otherwise $\sigma$ is called an essential half-saddle.

If a point $y$ in $Q$ is a maximum or minimum and lies on $\partial Q$, then $x$ is called a half-center.

Consider $h: Q \rightarrow R$ and $C \subset \partial Q$. We say $h$ is Morse on $Q$ rel $C$, if $h$ is Morse on $Q-C$, and near $C, h$ parametrizes a collar $C \times I$ in $Q$ of $C$, such that $h$ is constant on $C \times\{t\}$. This definition works equally well for manifolds of arbitrary dimension.

Let $Q$ be a compact surface. The saddle $\sigma$ is an outermost essential saddle above (below) $Q$ if 


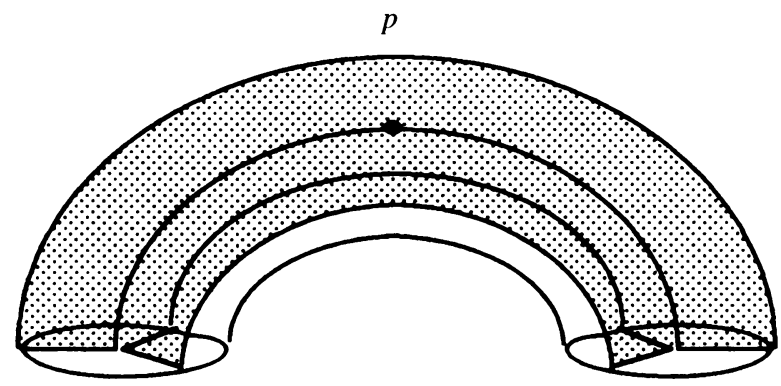

Neighborhood of $e$

FIGURE 3.1

(i) all separatrices of $\sigma$ end on $\partial Q$

(ii) $\sigma$ cuts off two upper (lower) and one lower (upper) disk.

Note: In particular, (ii) implies that all separatrices of $\sigma$ end on the same component of $\partial Q$.

Let $e \epsilon E$. Let $\alpha$ be a simple arc in $P$ connecting $x=p(e)$ to $\partial P$. In the remainder of this section $A$ will denote the saturated annulus $p^{-1}(\alpha)$ which projects to $\alpha$. Note that $A$ is an immersed but not embedded annulus. We will consider the annulus $\hat{A}$ which is the preimage of this immersion. Let $\partial_{1} \hat{A}$ be the component of $\partial \hat{A}$ which is mapped into $\partial M$ and let $\partial_{2} \hat{A}=\partial \hat{A}-\partial_{1} \hat{A}$. Let $\partial_{i} A$ be the image of $\partial_{i} \hat{A}$.

Let $h$ be a Morse function corresponding to the Heegaard splitting defined by the splitting surface $F$. We may assume that $h$ is Morse on $M$ rel $\partial M$. The function $h$ restricts to a function on $A$, which is a Morse function rel $\partial_{1} \mathrm{~A}$ on $A-e$; this function pulls back to a function $\hat{h}$ on $\hat{A}$, which is a Morse function rel $\partial_{1} \hat{A}$ on $\hat{A}-\partial_{2} \hat{A}$, but does not extend to all of $\hat{A}$, since critical points of $\left.h\right|_{e}$ give rise to two or more critical points on $\partial_{2} \hat{A}$ occurring at the same level. But if we denote the set of preimages of critical points of $\left.h\right|_{e}$ by $S$, then $\left.\hat{h}\right|_{\hat{A}-S}$ is a Morse function rel $\partial_{1} \hat{A}$. We denote by $\Phi_{\hat{A}}$ the foliation of $\hat{A}$ with respect to $\hat{h}$.

Proposition 3.1. After isotopy of $A$ rel $\partial_{1} A, \Phi_{\hat{A}}$ contains an outermost essential saddle.

Lemma 3.2. After isotopy of $A$ rel $\partial_{1} A, \Phi_{\hat{A}}$ does not contain any half-saddles.

Proof. Since $h$ is a Morse function rel $\partial M, h$ takes on a maximal (minimal) value on a connected component $C$ of $\partial M$ and a strictly smaller (greater) value on any point in a collar of $C$ in $M$. Thus a half-saddle in $A$ cannot have its critical point on $C$ and hence must have its critical point on the exceptional fiber $e$. Consider a small closed regular neighborhood $N(e)$ of $e$. Let $\sigma$ be a half-saddle based at $p \in e$. Let $S$ be a regular neighborhood of $p$ in $M$ consisting of meridian disks of $N(e)$. See Figure 3.1.

Then $e$ has a maximum (minimum) at $p$, but some components of $A \cap S$ lie above (below) $p$ near $p$. Here $A \cap S$ consists of squares $A_{1}, \ldots, A_{k}$ ( $k$ is the index of $e$ ) with three sides on $\partial S$, and one on $e$. Around $p$ we may use the meridian disks comprising $S$ to isotope $A_{1}, \ldots, A_{k}$ to lie entirely below (above) $p$. The isotopy thus described replaces the half-saddle with a 


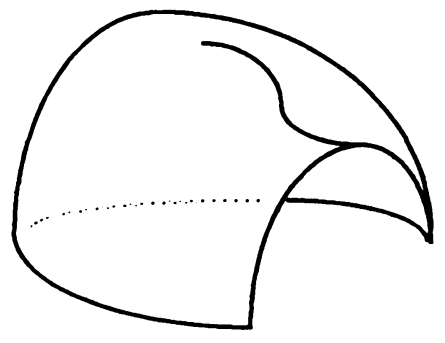

a half-saddle

FIGURE 3.2a

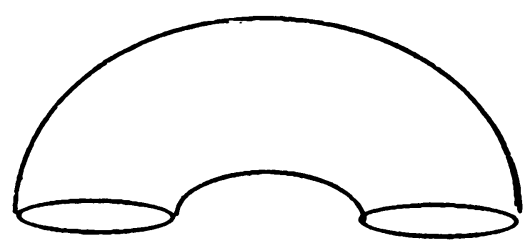

$P$

Figure 3.3a

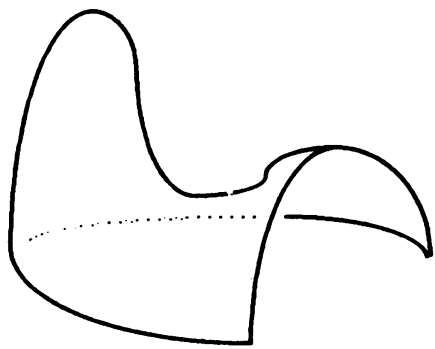

interior saddle point and half-center

FIGURE 3.2b

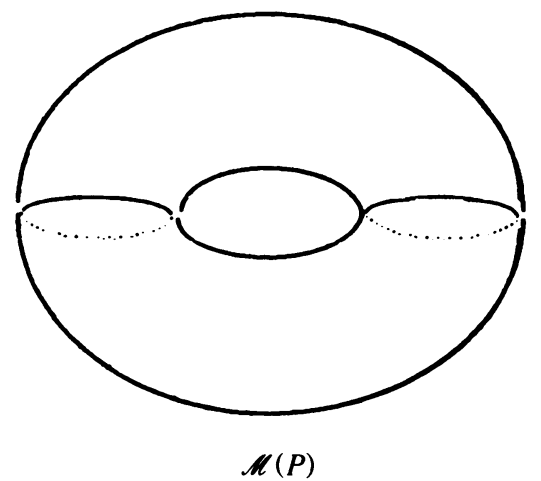

FIGURE 3.3b

half-center and an interior saddle point. It may be performed in an arbitrarily small neighborhood of $p$. See Figures 3.2a and 3.2b.

The content of Lemmas 3.3 and 3.4 is similar. Lemma 3.4 will be needed when $P$ is an annulus.

Lemma 3.3. Suppose $Q$ is a compact surface and $h: Q \rightarrow R$ is a Morse function rel $\partial Q$. Set $\epsilon(Q)=\max \{2-|\partial Q|, 0\}$ and let $\Phi$ be the singular foliation by level sets of $h$ on $Q$. Then at least $\epsilon(Q)-\chi(Q)$ leaves of $\Phi$ are essential saddles.

Proof. [12, 4.4].

Lemma 3.4. Let $P$ be a planar surface with at least two boundary components. Suppose that $\Phi_{P}$ is the foliation of $P$ with respect to a Morse function rel $\partial P$ such that $h(\partial P)$ is a constant. Then $\Phi_{P}$ contains an essential saddle. See Figures 3.3a and 3.3b.

Proof. We may assume that $h: P \rightarrow[0,1]$. Without loss of generality, $h(\partial P)=0$. This implies that $-h$ is also a Morse function rel $\partial P$ on $P$. Let $P^{\prime}$ be a copy of $P$ and consider the mirror double $\mathscr{M}(P)=\left(P \cup P^{\prime}\right) / \sim$ 


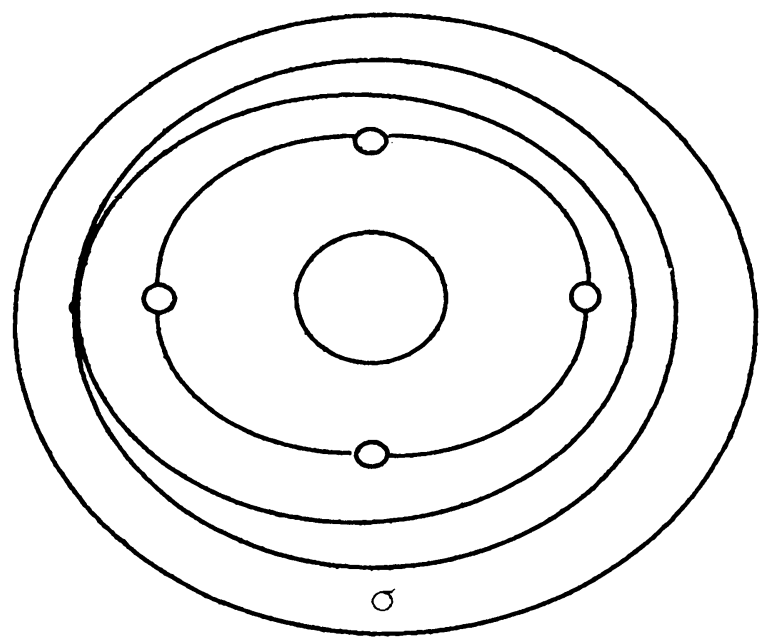

Figure 3.4a

of $P$, where $\sim$ identifies corresponding points in $\partial P$ and $\partial P^{\prime}$. Now $\mathscr{M}(P)$ is endowed with a Morse function $f$ obtained from $h$ on $P$ and $-h$ on $P^{\prime}$. This $f$ is a Morse function on the orientable surface $\mathscr{M}(P)$ of positive genus; and since the Euler characteristic of this surface is less than or equal to 0 , $\Phi_{\mathscr{M}(P)}$ contains at least two essential saddles, by Lemma 3.3. Since a bicollar of $[\partial P]$ is foliated by circles, these essential saddles cannot intersect $[\partial P]$, hence lie either entirely in $\Phi_{P}$ or $\Phi_{P^{\prime}}$. By symmetry, there must be at least one essential saddle in $\Phi_{P}$.

Lemma 3.5. If $\Phi_{\hat{A}}$ contains no half-saddles, then $\Phi_{\hat{A}}$ contains a saddle all of whose separatrices end on $\partial_{2} \hat{A}$.

Proof. Let $S$ be the set of critical points of $\left.h\right|_{e}$. Note that $|S| \geq 2$. Denote by $\hat{S}$ the set of critical points in $\hat{A}$ corresponding to $\hat{S}$. Then $|\hat{S}| \geq 4$. Each point in $\hat{S}$ is a half-center. Let $N(\hat{S})$ be a closed regular neighborhood of $\hat{S}$ in $\hat{A}$, such that $\hat{h}$ is constant on each component of $\partial N(\hat{S})$. Set $B=$ closure $(\hat{A}-N(\hat{S}))$.

Let $B^{\prime}$ be a copy of $B$ and $\left(\left.\hat{h}\right|_{B^{\prime}}\right)$ ' a copy of $\left.\hat{h}\right|_{B}$. Consider the double $\mathscr{D}(B)$ $=B \cup B^{\prime} / \sim$ of $B$, where $\sim$ identifies the points in $\partial_{2} B=\partial_{2} \hat{A} \cap \partial B$ with the corresponding points in $\partial B^{\prime}$. The function $f: \mathscr{D}(B) \rightarrow[0,1]$, defined by $\left(\left.\hat{h}\right|_{B^{\prime}}\right)^{\prime}$ on $B^{\prime}$ and $\left.\hat{h}\right|_{B}$ on $B$ is a Morse function rel $\partial \mathscr{D}(B)$ and will be called the Morse function induced on $\mathscr{D}(B)$. Here $\mathscr{D}(B)$ is a planar surface with $|\hat{S}|+2 \geq 6$ boundary components. By Lemma 3.3, $\Phi_{\mathscr{D}(B)}$, the foliation of the $\mathscr{D}(B)$ with respect to $f$, contains an essential saddle $\sigma$.

Since $\Phi_{\hat{A}}$ contains no half-saddles, $\left|\sigma \cap\left[\partial_{2} B\right]\right|$ is even and the saddle point $x$ lies either in $B$ or in $B^{\prime}$. We may assume that $x$ lies in $B$. We may further assume that $\sigma$ is an outermost essential saddle (which does not a priori mean that $\sigma$ is an outermost saddle above or below $\left.h^{-1}(\sigma)\right)$.

Case 1: $\left|\sigma \cap\left[\partial_{2} B\right]\right|=0$ (Figure 3.4a).

Then $\sigma \cap \partial_{2} \hat{A}=\varnothing$ and the separatrices combine in pairs to form circles parallel to $\partial_{2} \hat{A}$. Let $c$ be the circle closest to $\partial_{2} B$. We may consider the surface 


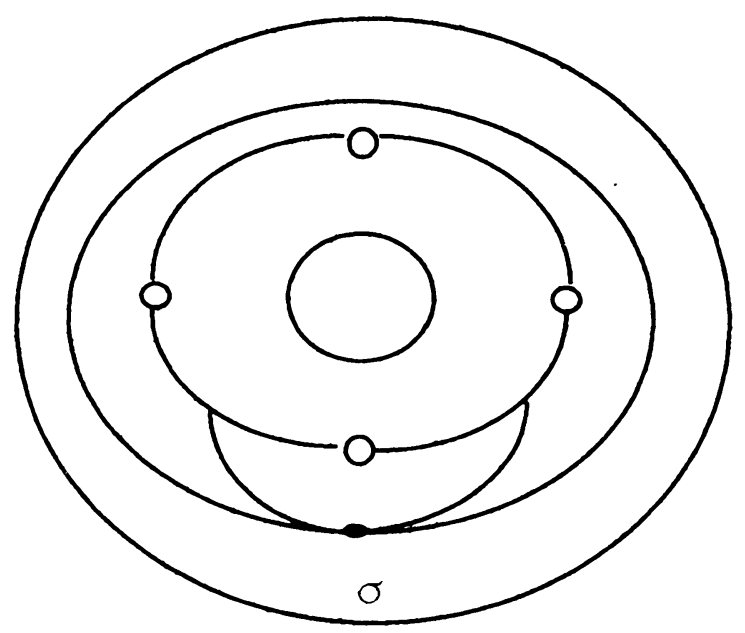

FIGURE 3.4b

$\tilde{B}$ obtained by cutting $B$ along $c$ and taking the component which contains $\partial_{2} B$. By the argument above, $\tilde{B}$ contains an essential saddle, contradicting the assumptions.

Case 2: $\left|\sigma \cap\left[\partial_{2} B\right]\right|=2$ (Figure 3.4b).

Since $\Phi_{\hat{A}}$ contains no half-saddles, the intersections occur in the interior of two of the separatrices. The other pair of separatrices combines to form a circle parallel to $\partial_{2} \hat{A}$, since $\hat{h}$ is constant on $\partial_{1} \hat{A}$. The leaf $\sigma$ cuts $B$ into one annulus and two disks $D_{1}, D_{2}$. Here $\partial D_{1}$ (respectively $\partial D_{2}$ ) consists of subarcs of $\sigma$ and of $\partial N(\hat{S})$ together with a collection $B_{1}$ (respectively $B_{2}$ ) of subarcs of $\partial_{2} B$; here $\partial_{2} B=B_{1} \cup \mathrm{B}_{2}$.

Claim: Either $B_{1}$ or $B_{2}$ contains at least four connected components.

We may consider the essential saddle $\sigma$ in $\hat{A}$. It cuts $\hat{A}$ into one annulus and two disks $\hat{D}_{1}$ and $\hat{D}_{2}$, corresponding to $D_{1}$ and $D_{2}$. Consequently $\partial \hat{D}_{1}$ $\left(\partial \hat{D}_{2}\right)$ consists of subarcs of $\sigma$ and an arc $b_{1}\left(b_{2}\right)$ in $\partial_{2} \hat{A}$; where $b_{1} \cup b_{2}=$ $\partial_{2} \hat{A}$. The number of connected components of $B_{1}\left(B_{2}\right)$ exceeds the number of critical points on $b_{1}\left(b_{2}\right)$ by exactly one. Since $e$ has index at least two, $\hat{h}$ attains its maximum (minimum) at least twice on $\partial_{2} \hat{A}$. Note that $b_{1}$ and $b_{2}$ must each contain an odd number of critical points. So either $b_{1}$ or $b_{2}$ contains at least three critical points. This proves the claim.

Without loss of generality $B_{1}$ contains at least four components. Let $l$ be the level set of $\left.h\right|_{\hat{A}}$ which contains $\sigma$. Cut $D_{1}$ along $l$ and consider the component $\tilde{D}_{1}$ whose boundary contains $\sigma \cap \partial D_{1}$.

Let $\mathscr{D}\left(\tilde{D}_{1}\right)$ be the double of $\tilde{D}_{1}$ along $\partial \tilde{D}_{1} \cap \partial_{1} B$. Then $\mathscr{D}\left(\tilde{D}_{1}\right)$ is a planar surface with at least two boundary components and the induced function on $\mathscr{D}\left(\tilde{D}_{1}\right)$ is a Morse function rel $\partial \mathscr{D}\left(\tilde{D}_{1}\right)$. An argument similar to the one above then shows that $\Phi_{\tilde{D}_{1}}$, the foliation of $\tilde{D}_{1}$ with respect to the induced Morse function rel $\partial \tilde{D}_{1}$, contains an essential saddle, again contradicting the assumptions.

Case 3: $\left|\sigma \cap\left[\partial_{2} B\right]\right|=4$ (Figure 3.4c). 


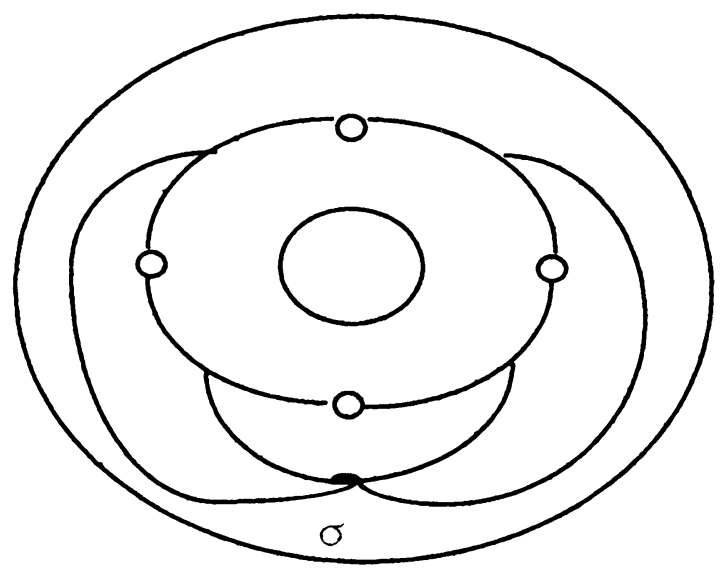

FIGURE 3.4C

Proof. (Proposition 3.1) By Lemma 3.2 and 3.5, $\Phi_{\hat{A}}$ contains, possibly after isotopy of $A$ rel $\partial A$, a saddle $\sigma$ all of whose separatrices end on $\partial_{2} \hat{A}$. We need only show that either $\sigma$ cuts off two lower and one upper disk or $\sigma$ cuts off two upper and one lower disk.

Suppose $\sigma$ is a saddle in $\Phi_{\hat{A}}$ all of whose separatrices end on $\partial_{2} \hat{A}$. If one of the disks $D$ cut off by $\sigma$ is not an upper or lower disk, then there is a leaf $\tau$ in $\Phi_{\hat{A}}$, which occurs at the same level as $\sigma$. Since $\tau$ occurs at the same level as $\sigma, \tau$ must be a regular leaf.

Case 1: $\tau$ is a simple closed curve.

Consider the component $\tilde{A}$ of $D$ cut along $\tau$ which contains subarcs of $\sigma$. Lemma 3.4 applied to the double $\mathscr{D}(\tilde{A})$ of $\tilde{A}$ along $\partial \tilde{A}-(\sigma \cup \tau)$ shows that $\Phi_{\tilde{A}}$ must contain an essential saddle $\sigma^{1}$.

Case 2: $\tau$ is an arc with endpoints on $\partial_{2} \hat{A}$.

Let $\tilde{D}$ be the component of $D$ cut along $\tau$ which contains subarcs of $\sigma$. Lemma 3.4 applied to the double $\mathscr{D}(\tilde{D})$ of $\tilde{D}$ along $\partial \tilde{D}-(\sigma \cup \tau)$ shows that $\Phi_{\tilde{D}}$ must contain an essential saddle $\sigma^{1}$.

An essential saddle in a disk has all separatrices ending on the boundary of that disk. In cases 1 and 2 above, this implies that the essential saddle $\sigma^{1}$ has separatrices ending on $\partial_{2} \hat{A}$. We see that if we choose $\sigma$ to be an outermost saddle with all separatrices ending on $\partial_{2} \hat{A}$, then neither case 1 nor case 2 can occur and hence $\sigma$ must cut off either two upper disks and one lower disk or one upper disk and two lower disks.

\section{The MAIN ARgument}

Definitions. Let $\alpha$ be a collection of closed curves in the manifold $M$ on which there is defined a Morse function $h: M \rightarrow[0,1]$. After an isotopy of $\alpha$ we may assume that $\left.h\right|_{\alpha}$ is a Morse function. Let $r_{0}, \ldots, r_{n}$ be the critical values of $\left.h\right|_{\alpha}$. Choose $l_{1}, \ldots, l_{n}$ so that $r_{0}<l_{1}<r_{1}<\ldots<l_{n}<r_{n}$. Let $L_{1}, \ldots, L_{n}$ be level surfaces of $h$ corresponding to $l_{1}, \ldots, l_{n}$. The number $\left|L_{1} \cap \alpha\right|+\ldots+\left|L_{n} \cap \alpha\right|$ is called the width of $\alpha$ with respect to $h$. We say 


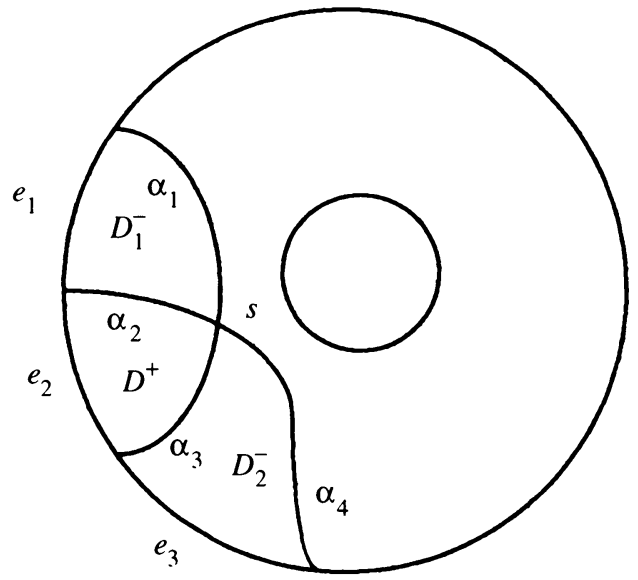

FIGURE 4.1. Labelling of the outermost essential saddle $\sigma$

that $\alpha$ is in thin position with respect to $h$ if the width of $\alpha$ with respect to $h$ is minimal.

For details see $[4, \S 4]$ or $[5, \S 1]$.

Lemma 4.1. Let $e$ be an exceptional fiber in $M$. Then $F$ is vertically reducible at $e$.

Proof. Let $e$ be an exceptional fiber of $M$. We may assume that, after a small isotopy, $e$ is disjoint from the the cores of the compression bodies in the Heegaard splitting of $M$. Thus after this small isotopy, $e$ intersects only level surfaces of $h$ which are isotopic to $F$. We may also assume that, after isotopy, $e$ is in thin position with respect to $h$.

Let $\beta$ be an arc in $P$ connecting $\partial P$ to $x=\pi(e)$. Let $A$ be the saturated annulus which projects to $\beta$. Let $\sigma$ be an outermost essential saddle above or below $\hat{A}$ in $\Phi_{\hat{A}}$ and let $L$ be the level surface of $h$ whose intersection with A induces $\sigma$. Without loss of generality, $\sigma$ is an outermost essential saddle below $A$, hence cuts off two lower disks, $D_{1}^{-}$and $\mathrm{D}_{2}^{-}$, and one upper disk, $D^{+}$.

Let $s$ be the critical point of $\sigma$. Let $\alpha_{2}$ be the subarc of $\partial D_{1}^{-}$shared by $\partial D^{+}, \alpha_{1}$ the subarc of $\partial D_{1}^{-}$connecting $\mathrm{s}$ to $\partial A$ which is not in $\partial D^{+}, \alpha_{3}$ the subarc of $\partial D_{2}^{-}$shared by $\partial D^{+}$and $\alpha_{4}$ the subarc of $\partial D_{2}^{-}$connecting $s$ to $\partial A$ which is not in $\partial D^{+}$. Denote by $p_{i}$ the endpoint of $\alpha_{i}$ on $e$. Further, let $e_{1}$ be the subarc of $e$ in $\partial D_{1}^{-}, e_{2}$ the subarc of $e$ in $\partial D^{+}$and $e_{3}$ the subarc of $e$ in $\partial D_{2}^{-}$. See Figure 4.1.

Set $\gamma_{1}=\alpha_{1} \cup \alpha_{3}, \gamma_{2}=\alpha_{2} \cup \alpha_{4}$ and parametrize $\gamma_{1}, \gamma_{2}$ as paths. In $A$, the oriented intersection number $I\left(\gamma_{1}, \gamma_{2}\right)= \pm 1$. Also in $L$, the oriented intersection number $I\left(\gamma_{1}, \gamma_{2}\right)= \pm 1$. (The signs may differ when we look at the oriented intersection number in $A$ versus $L$.) See Figure 4.2.

Claim 1: The curves $\gamma_{1}, \gamma_{2}$ are closed curves based at points $p_{1}, p_{2}$ on $e$ (i.e., $p_{1}=p_{3}$ and $p_{2}=p_{4}$ ). In particular, $e_{1}=e_{3}$ as subarcs of $e$.

Since $L$ is both high and low with respect to $A$, it follows from the proof of [5, Lemma 2.1] or $[4, \S 4]$ that $p_{1}=p_{3}$ and $p_{2}=p_{4}$. Since $e_{1}, e_{3}$ contain 


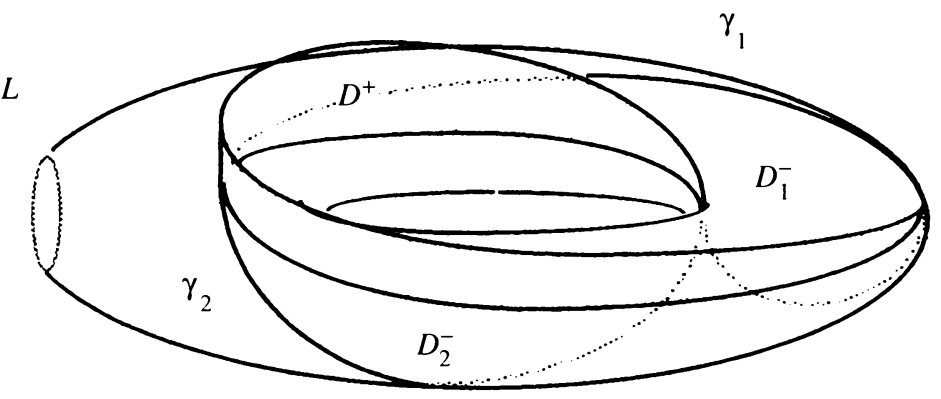

FIGURE 4.2. $L, D_{1}$ and $D_{2}$ in $M$

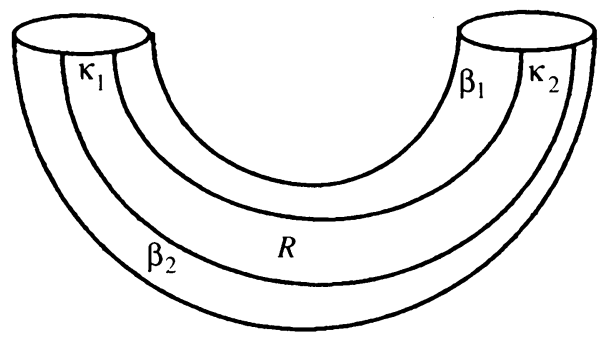

FIGURE 4.3. The arcs $\beta_{1}, \beta_{3}, \kappa_{1}$ and $\kappa_{2}$

the minimum of $e$ and $e_{2}$ contains the maximum of $e$, the claim follows.

Claim 1 implies that $e_{1} \cup e_{2}=e$. Hence $D_{1}^{-} \cup D^{+}$defines an isotopy of $\gamma_{1}$ and $e$. This fact will be used later.

Now $s=\gamma_{1} \cap \gamma_{2}$. Set $\tilde{A}=A-\eta(e), \tilde{D}_{1}^{-}=D_{1}^{-} \cap \tilde{A}, \tilde{D}_{2}^{-}=D_{2}^{-} \cap$ $\tilde{A}, \tilde{\alpha}_{1}=\alpha_{1} \cap \tilde{A}, \ldots, \tilde{\alpha}_{4}=\alpha_{4} \cap \tilde{A}$. Label the endpoint of $\tilde{\alpha}_{i}$ on $\partial \tilde{A}$ by $q_{i}$, and the subarc of $\partial \tilde{A}$ connecting $q_{i}$ to $q_{i+1}$ by $\beta_{i}$.

Since $L$ is an orientable surface, a copy $\tilde{\gamma}_{1}$ of $\gamma_{1}$ can be isotoped, in $L$, off of $\gamma_{1}$ to one side (called the outside, the other side being called the inside; note that since $\gamma_{1}$ may be nonseparating in $L$, this terminology only makes sense locally). This isotopy can be performed in the complement of $\gamma_{2}$, except near $s$, where $\tilde{\gamma}_{1}$ will intersect $\gamma_{2}$ exactly once. So $I\left(\tilde{\gamma}_{1}, \gamma_{2}\right)= \pm 1$ persists.

Since $e$ intersects $L$ in exactly two points $p_{1}, p_{2}, L \cap N(e)$ consists of exactly two disks $P_{1}$ and $P_{2}$, for $N(e)$ small enough. Now $\beta_{1}$ and $\beta_{3}$ are parallel arcs on $\partial N(e)$, with one endpoint on $\partial P_{1}$ and the other on $\partial P_{2}$. They both lie below $L$. On the other hand, $\beta_{2}$ is an $\operatorname{arc}$ in $\partial N(e)$, with one endpoint on $\partial P_{1}$ and the other on $\partial P_{2}$, which lies above $L$. Note that $\beta_{1}$ and $\beta_{3}$ are parallel in $N(e)$ to $e_{1}$, and $\beta_{2}$ is parallel in $N(e)$ to $e_{2}$. In particular, in the annulus of $\partial N(e)$ below $L, \beta_{1}$ and $\beta_{3}$ cut off two rectangles with corners $q_{1}$, $q_{2}, q_{3}, q_{4}$. Let $R$ be the rectangle with sides $\beta_{1}, \beta_{3}, \kappa_{1}$ and $\kappa_{2}$, where $\kappa_{1}$ is chosen to lie on the inside of $\gamma_{1}$ and is hence disjoint from $\tilde{\gamma}_{1}$. The choice of $\kappa_{1}$ determines $\kappa_{2}$. See Figure 4.3.

Claim 2: $\tilde{D}_{1}^{-}$may be altered slightly, so as to be disjoint from $\tilde{D}_{2}^{-}$. Furthermore, this alteration can be made away from $\tilde{\gamma}_{1}$.

Let $N(s)$ be a small 3-ball neighborhood of $s$ in $M$ missing $\tilde{\gamma}_{1}$. We replace 
the portion of $\tilde{D}_{1}^{-}$which lies in $\operatorname{int}(N(s))$ by a disk in $\partial N(s)$. More specifically, $\partial N(s) \cap \tilde{D}_{1}^{-}$contains an arc $\tau_{1}$ which together with an arc $\tau_{2}$ in $L \cap$ $\partial N(s)$ bounds a disk $D^{\prime}$ in $N(s)$. Replace $\tilde{D}_{1}^{-}$by $\hat{D}_{1}=\left(\tilde{D}_{1}^{-}-\operatorname{int}(N(s))\right) \cup D^{\prime}$.

Set $D^{-}=\hat{D}_{1}^{-} \cup \tilde{D}_{2}^{-} \cup R$. Then since the above alteration was made away from $\tilde{\gamma}_{1}, \partial D^{-} \cap \tilde{\gamma}_{1}=\left(\begin{array}{lll}\gamma_{1} \cup \gamma_{2}\end{array}\right) \cap \tilde{\gamma}_{1}=\gamma_{2} \cap \tilde{\gamma}_{1}$. Thus $I\left(\partial D^{-}, \tilde{\gamma}_{1}\right)=$ \pm 1 . In particular, this implies that $\partial D^{-}$is essential and thus that $D^{-}$is an essential disk. Now $D^{-}$is a lower disk for $L$ such that $\partial D^{-}$intersects $\tilde{\gamma}_{1}$ once transversely. Since $L$ is isotopic to $F$ and $e$ is isotopic to $\tilde{\gamma}_{1}$, the result now follows from Lemma 2.2.

Theorem 4.2. Every irreducible Heegaard splitting of a fiberwise orientable Seifert fibered space with nonempty boundary is vertical.

Proof. Let $M$ be a Seifert fibered space with nonempty boundary. The proof follows by induction on the number or exceptional fibers in $M$. Theorem 1.1 establishes the result in the case that $M$ has no exceptional fibers. Now suppose that the result holds for all Seifert fibered spaces with nonempty boundary and at most $n$ exceptional fibers and that $M$ has $n+1$ exceptional fibers. Consider the splitting surface $F$ of an irreducible Heegaard splitting for $M$. Let $e$ be an exceptional fiber of $M$. By Lemma 4.1 $F$ is vertically reducible at $e$. This means that, perhaps after isotopy, $F$ is also the splitting surface of a Heegaard splitting for $M-\eta(e)$. Since $M-\eta(e)$ has only $n$ exceptional fibers $F$ is a vertical Heegaard splitting for $M-\eta(e)$ (irreducibility of a Heegaard splitting in $M$ guarantees irreducibility of the corresponding Heegaard splitting in $M-\eta(e))$. It now follows from the construction that the Heegaard splitting of $M$ with splitting surface $F$ is vertical.

\section{The classification of Vertical HeEgaArd splittings of Seifert FIBERED SPACES AFTER LUSTIG AND MORIAH}

Given the verticality of irreducible Heegaard splittings of fiberwise orientable Seifert manifolds with boundary, it is natural to ask when two vertical Heegaard splittings are equivalent. This question was answered, in most cases, for closed Seifert fibered spaces in [7]. The content of this section is to tailor Theorem 2.8 in [7] to the class of manifolds under consideration. Lustig and Moriah define an invariant for Heegaard splittings of a closed 3-manifold $M$, by considering the handlebodies in a Heegaard splitting, and the Nielsen equivalence classes for generating systems of the fundamental group of $M$ which they define. This invariant serves to distinguish vertical Heegaard splittings of Seifert fibered spaces in most cases.

Theorem 5.1. Let $\beta=\left\{i \mid \beta_{i} \neq \pm 1\left(\bmod \alpha_{i}\right) ; i=1, \ldots, k\right\}$. Then the two vertical Heegaard splittings $F\left(i_{1}, \ldots, i_{n} ; j_{1}, \ldots, j_{l}\right)$ and $F\left(\zeta_{1}, \ldots, \zeta_{\nu} ; j_{1}, \ldots, j_{l}\right)$ are isotopic if and only if $\beta \cap\left\{i_{1}, \ldots, i_{n}\right\}=\beta \cap$ $\left\{\zeta_{1}, \ldots, \zeta_{\nu}\right\}$.

Remark 5.2. Consider the Heegaard splittings $\left(W_{1}, W_{2}\right)$ and $\left(W_{1}^{\prime}, W_{2}^{\prime}\right)$ of the 3-manifold $M$ with splitting surfaces $F$ and $F^{\prime}$. Suppose $F$ and $F^{\prime}$ are isotopic under an isotopy taking $W_{1}$ to $W_{1}^{\prime}$. Then $\partial_{-} W_{1}=\partial_{-} W_{1}^{\prime}$. 
Definition. Let $x=\left\{x_{1}, \ldots, x_{n}\right\}$ be a generating system for a group $G$. The generating system defines a canonical epimorphism $p_{x}$ of the free group $F=F\left(X_{1}, \ldots, X_{n}\right)$ onto $G$ by mapping $X_{i}$ to $x_{i}$. Two generating systems $\left(x_{1}, \ldots, x_{n}\right)$ and $\left(y_{1}, \ldots, y_{n}\right)$ of $G$ are Nielsen equivalent if there exists an isomorphism $g$, for which the following diagram commutes:

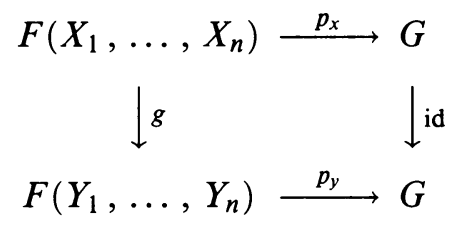

Remark 5.3. The generators of the fundamental group of a handlebody $H$ in a Heegaard splitting of a closed manifold $M$ define a Nielsen equivalence class $\mathscr{N}\left(\pi_{1}(M) ; H\right)$ of generators for $\pi_{1}(M)$. This assignment is unaffected by isotopies of $H$ and hence well defined on an equivalence class of Heegaard splittings. In order to define $\mathcal{N}\left(\pi_{1}(M) ; H\right), H$ need not be one of the handlebodies in a Heegaard splitting. The only requirement is that its inclusion induce an epimorphism of fundamental groups $i_{*}: \pi_{1}(H) \rightarrow \pi_{1}(M)$.

For general facts, such as these, on Nielsen equivalence as pertaining to equivalence classes of Heegaard splittings, see the remarks in [7, Definition 2.8]. (Note the relevance of [15, Theorem 5.8.3].) The two handlebodies in a Heegaard splitting $\left(H_{1}, H_{2}\right)$ of $M$ may define two different Nielsen equivalence classes $\mathcal{N}\left(\pi_{1}(M) ; H_{1}\right), \mathcal{N}\left(\pi_{1}(M) ; H_{2}\right)$. For $M$ a merely compact manifold, the assignment of a Nielsen equivalence class of generating systems to a Heegaard splitting of $M$ is less straightforward, since the fundamental group of a compression body is not free.

Remark 5.4. The vertical Heegaard splitting $\left(W_{1}\left(i_{1}, \ldots, i_{n} ; j_{1}, \ldots, j_{l}\right)\right.$, $\left.W_{2}\left(i_{1}, \ldots, i_{n} ; j_{1}, \ldots, j_{l}\right)\right)$ of the fiberwise orientable Seifert fibered space $M$ yields a natural generating system for $\pi_{1}(M) /\langle h\rangle$, where $\langle h\rangle$ is the subgroup of $\pi_{1}(M)$ generated by a regular fiber.

For recall the notation used in the introduction to define

$$
\left(W_{1}\left(i_{1}, \ldots, i_{n} ; j_{1}, \ldots, j_{l}\right), W_{2}\left(i_{1}, \ldots, i_{n} ; j_{1}, \ldots, j_{l}\right)\right) .
$$

Parametrize $a_{1}, b_{1}, \ldots, a_{g}, b_{g}$ as paths based at $x_{0}$. Let $\mathfrak{c}^{j}$ (resp. $\mathfrak{d}_{i}$ ) be a parametrization of $c^{j} \cup C^{j}$ (resp. $d_{i} \cup \partial D_{i}$ ) as a path based at $x_{0}$. And let $\tilde{\mathfrak{d}}_{i}$ denote the image of $e_{i}$, suitably parametrized under $i_{*}: \pi_{1}\left(V_{i}\right) \rightarrow$ $\pi_{1}\left(W_{1}, x_{0}\right)$. Then $\pi_{1}\left(W_{1}\right)$ is generated by (the homotopy classes of ) $a_{1}, b_{1}$, $\ldots, a_{g}, b_{g}, \mathfrak{c}^{2}, \ldots, \mathfrak{c}^{m}, \tilde{d}_{i_{1}}, \ldots, \mathfrak{d}_{i_{n}}, \mathfrak{d}_{l_{1}}, \ldots, \mathfrak{d}_{l_{k-n}}$ together with $l$ more generators (one per connected component of $\partial_{-} W_{1}$ ) each of which has $\langle h\rangle$ as its image under $i_{*}: \pi_{1}\left(W_{1}\right) \rightarrow \pi_{1}(M)$.

Now

$$
\begin{aligned}
\pi_{1}(M)=\left\langle a_{1}, b_{1}, \ldots,\right. & a_{g}, b_{g}, \mathfrak{c}^{2}, \ldots, \mathfrak{c}^{m}, \mathfrak{d}_{1}, \ldots, \mathfrak{d}_{k}, h \mid \\
& {\left[a_{i}, h\right](i=1, \ldots, g),\left[b_{i}, h\right](i=1, \ldots, g), } \\
{\left[\mathfrak{c}^{j}, h\right](j=2, \ldots, m),\left[\mathfrak{d}_{l}, h\right](l=1, \ldots, k), } & \left.\mathfrak{d}_{l}^{\alpha_{l}} h^{-\beta_{l}}(l=1, \ldots, k)\right\rangle .
\end{aligned}
$$


So

$$
\begin{aligned}
\pi_{1}(M) /\langle h\rangle=\left\langle a_{1}, b_{1}, \ldots, a_{g}, b_{g}, \mathfrak{c}^{2}, \ldots, \mathfrak{c}^{m},\right. \\
\mathfrak{d}_{1}, \ldots, \mathfrak{d}_{k}(l=1, \ldots, k)\left|\mathfrak{d}_{l}^{\alpha_{l}}\right\rangle .
\end{aligned}
$$

To find the natural generating system for $\pi_{1}(M) /\langle h\rangle$ determined by $W_{1}\left(i_{1}, \ldots, i_{n} ; j_{1}, \ldots, j_{l}\right)$ we thus need only compute the image of $\tilde{d}_{i_{1}}, \ldots, \tilde{d}_{i_{n}}$ under the composition of maps

$$
\pi_{1}\left(W_{1}\right) \stackrel{i_{*}}{\longrightarrow} \pi_{1}(M) \rightarrow \pi_{1}(M) /\langle h\rangle .
$$

This computation is made in [7, Lemma 2.7]. The resulting generators for $\pi_{1}(M) /\langle h\rangle$ are $\mathfrak{d}_{i_{1}}^{-\gamma_{i_{1}}}, \ldots, \mathfrak{d}_{i_{n}}^{-\gamma_{i_{n}}}$, where $\gamma_{i_{j}}$ is such that if $\left(\alpha_{i_{j}}, \beta_{i_{j}}\right)$ are the invariants associated with $e_{i_{j}}$, then for some $\delta_{i_{j}}, \beta_{i_{j}} \gamma_{i_{j}}-\alpha_{i_{j}} \delta_{i_{j}}=1$ and $1 \leq \gamma_{i_{j}}<\alpha_{i_{j}}$. The natural generating system for $\pi_{1}(M)$ determined by $W_{1}\left(i_{1}, \ldots, i_{n} ; j_{1}, \ldots, j_{l}\right)$ is thus $\left\{a_{1}, b_{1}, \ldots, a_{g}, b_{g}, \mathfrak{c}^{2}, \ldots, \mathfrak{c}^{m}, \tilde{d}_{i_{1}}{ }^{-\gamma_{i_{1}}}\right.$, $\left.\ldots, \tilde{\mathfrak{d}}_{i_{n}}-\gamma_{i_{n}}, \mathfrak{d}_{l_{1}}, \ldots, \mathfrak{d}_{l_{k-n}}\right\}$.

Note that this generating system has the same rank as $\pi_{1}(M) /\langle h\rangle$. Also notice that since $\beta_{i_{j}} \leq \alpha_{i_{j}}$ the equality $\beta_{i_{j}} \gamma_{i_{j}}-\alpha_{i_{j}} \delta_{i_{j}}=1$ implies $\gamma_{i_{j}}>1$.

Remark 5.5. Two generating systems

$$
u=x_{1}^{u_{1}}, \ldots, x_{n}^{u_{n}}, x_{n+1}, \ldots, x_{k}
$$

and

$$
v=x_{1}^{v_{1}}, \ldots, x_{n}^{v_{n}}, x_{n+1}, \ldots, x_{k}
$$

in the free product $G$ of a finite number of cyclic groups,

$$
G=\left\langle x_{1}, \ldots, x_{n}, x_{n+1}, \ldots, x_{k} \mid x_{1}^{\alpha_{1}}, \ldots, x_{n}^{\alpha_{n}}\right\rangle
$$

are Nielsen equivalent if and only if $u_{i}= \pm v_{i} \bmod \alpha_{i}$ for all $i=1, \ldots, n$.

This is a consequence of [6, Theorem 1] and Grushko's Theorem.

Lemma 5.6. If $\beta_{i_{n}}= \pm 1\left(\bmod \alpha_{n}\right)$, then

$$
F\left(i_{1}, \ldots, i_{n} ; j_{1}, \ldots, j_{l}\right)
$$

and

$$
F\left(i_{1}, \ldots, i_{n-1} ; j_{1}, \ldots, j_{l}\right)
$$

are isotopic.

Proof. See [7, Theorem 2.8].

Definitions. Let $W$ be a compression body. Then $W$ is obtained from $\partial_{-} W \times$ $I$ by attaching 1-handles to $\partial_{-} W \times\{1\}$. Suppose $x$ is a point in $\partial_{-} W \times\{1\}$ disjoint from the attaching disks of the 1-handles. Then the proper spanning arc

$$
\{x\} \times I \subset \partial_{-} W \times I \subset W
$$

is called the vertical arc based at $\{x\} \times\{0\}$ in $\partial_{-} W$.

Let $C^{1}, \ldots, C^{n}$ be the components of $\partial_{-} W$ and let $\Gamma=\left\{\gamma^{1}, \ldots, \gamma^{n}\right\}$ be a collection of vertical arcs such that each $\gamma^{j}$ is a vertical arc based at a point in $C^{j}$. We call $\Gamma$ a vertical collection of spanning arcs for $W$. Since $W-\Gamma$ is a handlebody, the inclusion $i:(W-\Gamma) \rightarrow W$ defines a Nielsen equivalence 
class of generating systems for $\pi_{1}(W)$ via $i_{*}: \pi_{1}(W-\Gamma) \rightarrow \pi_{1}(W)$. We call this class the Nielsen class of $\pi_{1}(W)$ determined by $\Gamma$.

Two properly isotopic classes $\Gamma$ and $\Gamma^{\prime}$ of vertical spanning arcs for $W$ determine the same Nielsen class of $\pi_{1}(W)$. Indeed, the isotopy of $\Gamma$ and $\Gamma^{\prime}$ determines an isotopy of the handlebodies $H=W-\Gamma$ and $H=W-\Gamma^{\prime}$; the Nielsen class of $\pi_{1}(W)$ determined by $\Gamma$ is $\mathcal{N}\left(\pi_{1}(W) ; H\right)$ and the Nielsen class of $\pi_{1}(W)$ determined by $\Gamma^{\prime}$ is $\mathscr{N}\left(\pi_{1}(M) ; H^{\prime}\right)$, thus they are equivalent by Remark 5.3. In particular, for any two vertical collections of spanning arcs $\Gamma$ and $\Gamma^{\prime}$, the Nielsen class of $\pi_{1}(W)$ determined by $\Gamma$ coincides with the Nielsen class of $\pi_{1}(\mathrm{~W})$ determined by $\Gamma^{\prime}$, so long as both collections are vertical with respect to the same product structure.

Proof (Theorem 5.1). We first prove the following:

Claim: Suppose $W$ and $W^{\prime}$ are compression bodies in $M$, with $\left(W, \partial_{-} W\right)$ $\subset(M, \partial M)$ and $\left(W^{\prime}, \partial_{-} W^{\prime}\right) \subset(M, \partial M)$, and such that $i_{*}: \pi_{1}(W) \rightarrow \pi_{1}(M)$ and $i_{*}^{\prime}: \pi_{1}\left(W^{\prime}\right) \rightarrow \pi_{1}(M)$ are epimorphisms. Further suppose that $G: M \times$ $I \rightarrow M$ is a proper isotopy which takes $W$ to $W^{\prime}$. Let $\Gamma$ be a vertical collection of spanning arcs for $\mathrm{W}$ and let $\Gamma^{\prime}$ be a vertical collection of spanning arcs for $W^{\prime}$. Then $\mathscr{N}\left(\pi_{1}(M) ; W-\eta(\Gamma)\right)=\mathscr{N}\left(\pi_{1}(M) ; W^{\prime}-\eta\left(\Gamma^{\prime}\right)\right)$.

Denote by $g_{t}: M \rightarrow M$ the function defined by $g_{t}(x)=G(x, t)$. Loosely speaking we have $\partial_{-} W \times I \subset W \subset M$ and $\partial_{-} W^{\prime} \times I \subset W^{\prime} \subset M$. But the product structures may differ. To be precise, we have two homeomorphisms pr $:\left(\partial_{-} W \times I\right) \rightarrow(W-(1$-handles $))$ and $\operatorname{pr}^{\prime}:\left(\partial_{-} W^{\prime} \times I\right)$ $\rightarrow\left(W^{\prime}-(1\right.$-handles $\left.)\right)$ (where $\partial_{-} W=\partial_{-} W^{\prime}$, we will denote the components of $\partial_{-} W=\partial_{-} W^{\prime}$ by $\left.C^{1}, \ldots, C^{m}\right)$. Without loss of generality, $\left.\mathrm{pr}\right|_{\partial_{-} W \times\{0\}}$ and $\left.\mathrm{pr}^{\prime}\right|_{\partial_{-} W \times\{0\}}$ are projections onto the first coordinate. We must show that $W-\eta(\Gamma)$ and $W^{\prime}-\eta\left(\Gamma^{\prime}\right)$ are isotopic handlebodies. It suffices to show that $g_{1}(\Gamma)$ and $\Gamma^{\prime}$ are properly isotopic in $W^{\prime}$. We may consider the connected components of $\Gamma$ (resp. $\Gamma^{\prime}$ ) individually. It then suffices to show that for each $j, g_{1}\left(\gamma^{j}\right)$ is properly isotopic to $\left(\gamma^{j}\right)^{\prime}$ by an isotopy which fixes the complement in $M$ of $\eta\left(C^{j}\right)$.

Suppose $\gamma^{j}$ is based at $\{x\} \times\{0\}$ in $C^{j} \times\{0\}=\operatorname{pr}\left(C^{j} \times\{0\}\right)$ and that $\left(\gamma^{j}\right)^{\prime}$ is based at $\left\{x^{\prime}\right\} \times\{0\}$ in $\left(C^{j}\right) \times\{0\}=\operatorname{pr}^{\prime}\left(C^{j} \times\{0\}\right)$. Without loss of generality, $g_{1}(\operatorname{pr}(x \times\{1\}))$ is disjoint from the attaching disks for the 1-handles in $W^{\prime}$. Let $\alpha$ and $\beta$ be two curves in $C^{j}$ such that

(1) $\alpha \cap \beta=x$,

(2) $x^{\prime}$ lies on $\alpha$,

(3) $\operatorname{pr}(\alpha \times 1), \operatorname{pr}(\beta \times 1)$ are disjoint from the attaching disks for $W$ and $\operatorname{pr}^{\prime}(\alpha \times 1), \operatorname{pr}^{\prime}(\beta \times 1)$ are disjoint from the attaching disks for $W^{\prime}$. (See Figure 5.1.)

Set $A=\operatorname{pr}(\alpha \times I), B=\operatorname{pr}(\beta \times I), A^{\prime}=\operatorname{pr}^{\prime}(\alpha \times I), \tilde{A}=g_{1}(A)$ and $\tilde{B}=g_{1}(B)$. Now $g_{1}\left(\gamma^{j}\right)=\tilde{A} \cap \tilde{B}$ and thus lies in $\tilde{A}$. On the other hand, $\left(\gamma^{j}\right)^{\prime}$ lies in $A^{\prime}$. Since $\left.g_{1}\right|_{C^{\prime} \times\{0\}}$ is isotopic to the identity, $g_{1}(\beta)$ is isotopic to $\beta$ and hence has the same intersection number, namely 1 , with $\alpha$. Thus the 1-manifold $A^{\prime} \cap \tilde{B}$ has an odd number of ends on $C^{j} \times\{0\}$ and hence contains a spanning arc $\tilde{\gamma}^{j}$. Since $g_{1}\left(\gamma^{j}\right)$ and $\tilde{\gamma}^{j}$ are both spanning arcs in $\tilde{B}$ they are properly isotopic; since $\tilde{\gamma}^{j}$ and $\left(\gamma^{j}\right)^{\prime}$ are both spanning arcs in $A^{\prime}$ they are properly isotopic. This establishes the claim. 


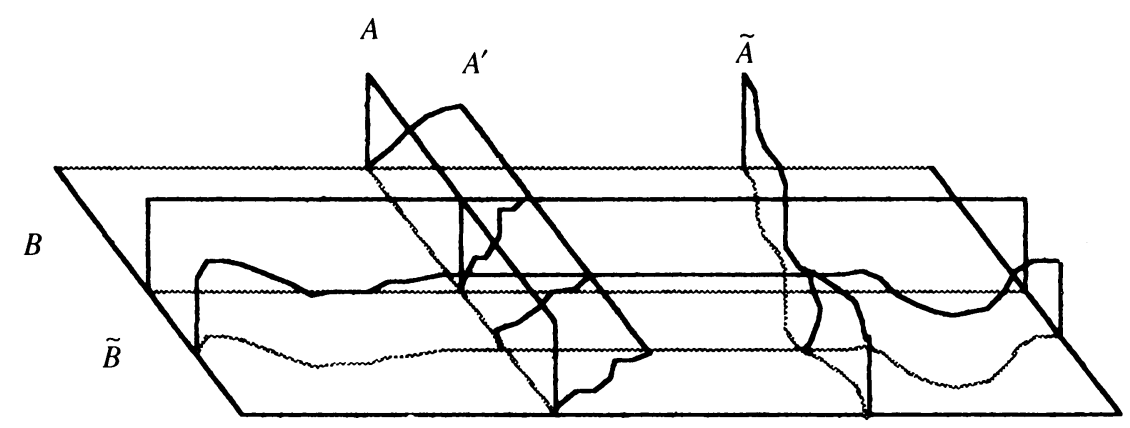

Figure 5.1. Partial depiction of the annuli $A, A^{\prime}, \tilde{A}, B$ and $\tilde{B}$

We will refer to the Nielsen equivalence class of generating systems for $\pi_{1}(M)$ provided by $W$ as in the claim by $\mathscr{N}\left(\pi_{1}(M) ; W\right)$ (i.e., $\mathscr{N}\left(\pi_{1}(M) ; W\right)=$ $\mathscr{N}\left(\pi_{1}(M) ; W-\Gamma\right)$, where $\Gamma$ is a vertical collection of spanning arcs for $W$ ). The claim also provides a Nielsen equivalence class of generating systems for $\pi_{1}(M) /\langle h\rangle$ determined by $W$, which we denote by $\mathscr{N}\left(\pi_{1}(M) /\langle h\rangle ; W\right)$. The generating system calculated in Remark 5.4 is in

$$
\mathscr{N}\left(\pi_{1}(M) /\langle h\rangle ; W\left(i_{1}, \ldots, i_{n} ; j_{1}, \ldots, j_{l}\right)\right) .
$$

Thus to show that two vertical Heegaard splittings are inequivalent, it suffices to show that

$$
\begin{aligned}
& \mathscr{N}\left(\pi_{1}(M) /\langle h\rangle ; W\left(i_{1}, \ldots, i_{n} ; j_{1}, \ldots, j_{l}\right)\right) \\
& \quad \neq \mathscr{N}\left(\pi_{1}(M) /\langle h\rangle ; W\left(\zeta_{1}, \ldots, \zeta_{\nu} ; j_{1}, \ldots, j_{l}\right)\right) .
\end{aligned}
$$

The theorem now follows from Proposition 1.2, Remarks 5.4 and 5.5 and Lemma 5.6.

\section{REFERENCES}

1. M. Boileau, D.J. Collins, and H. Zieschang, Genus 2 Heegaard decompositions of small Seifert manifolds, IHES/M/89/27

2. M. Boileau and J.P. Otal, Groupe des diffeotopies de certaines varietes de Seifert, C. R. Acad. Sci. Paris Ser. 1303 (1986).

3. A. Casson and C. Gordon, Reducing Heegaard splittings, Topology Appl. 27 (1987), 275283.

4. D. Gabai, Foliations and the topology of 3-manifolds III, J. Differential Geometry 26 (1987), 479-536.

5. C. Gordon and J. Luecke, Knots are determined by their complement, J. Amer. Math. Soc. 2 (1989).

6. M. Lustig, Nielsen equivalence and simple-homotopy type, Proc. London Math. Soc. (3) 62 (1991), 537-562.

7. M. Lustig and Y. Moriah, Nielsen equivalence in Fuchsian groups and Seifert fibered spaces, Topology 30 (1991), pp. 191-204.

8. Yoav Moriah, Heegaard splittings of Seifert fibered spaces, Invent. Math. 91 (1988), 465-481.

9. John Milnor, Morse theory, Lecture Notes by M. Spivak and R. Wells, Ann. of Math. Stud., no. 51, Princeton Univ. Press.

10. _ Lectures on the h-cobordism theorem, Notes by L. Siebenmann and J. Sondow, Princeton Math. Notes, Princeton Univ. Press, 1965 
11. M. Scharlemann and A. Thompson, Heegaard splittings of (surface) $\times I$ are standard, Math. Ann. 295 (1993), 549-564.

12. J. Schultens, The classification of Heegaard splittings for (closed orientable surface) $\times S^{1}$, Proc. London Math. Soc. (3) 67 (1993), 425-448.

13. P. Scott, The eight geometries of 3-manifolds, Bull. London Math. Soc. 15 (1983), 401-487.

14. H. Seifert, Topologie dreidimensionaler gefaserter Räume, Acta Math. 60 (1933), 147-238. Translation by W. Heil, memo notes, Florida State University, 1976.

15. H. Zieschang, E. Vogt, and H.D. Coldewey, Surfaces and planar discontinuous groups, Lecture Notes in Math., vol. 835, Springer-Verlag, 1988.

Department of Mathematics and Computer Science, Emory University, Fishburne Building, Atlanta, Georgia 30322

E-mail address: jcs@mathcs .emory.edu 